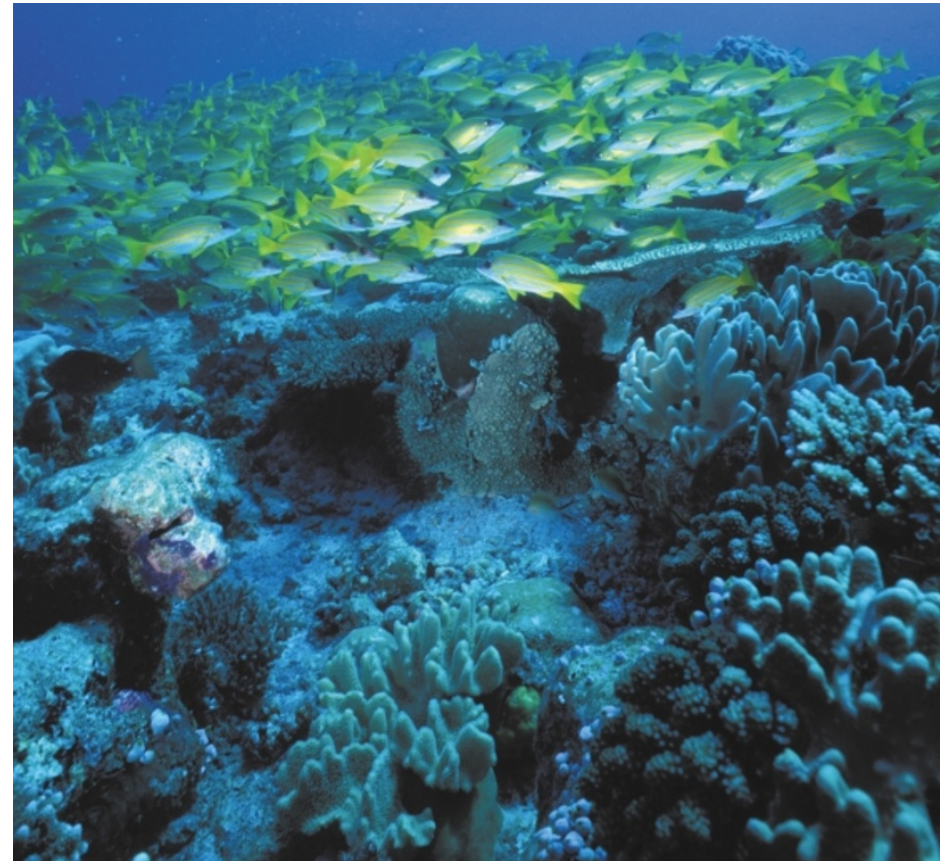

ECOLOGICAL GENETICS

\section{The secret to coral diversity}

Even if you don't know much about coral reefs, you're probably aware that they're amazingly beautiful and that the habitat of many is under threat. However, what makes corals so attractive in the first place - their incredible range of colours, shapes and sizes - is also proving to be a thorn in the side of conservation biologists, who struggle to distinguish one species from another. In fact, it's surprising that so many distinct forms exist at all. Corals are sexually very promiscuous: they reproduce by mass spawning and hybridize with each other, both factors that you expect would blur species boundaries. So, just how many species are there?

Steven Vollmer and Stephen Palumbi have now resolved this apparent paradox with regard to three Caribbean species. Using the most extensive genetic analysis done so far on corals, they have shown that instead of there being three coexisting species, there are in fact only two, which hybridize to form the third. Their methods should be applicable to resolve other coral species, and should help to inform conservation programmes.

The authors focused their attention on three morphologically distinct forms of Acropora corals: the branched 'staghorn' coral A. cervicornis; the flatter 'elkhorn' coral A. palmata; and the morphologically intermediate
A. prolifera. Intronic DNA sequence from two nuclear loci showed that the first two species are genetically distinct, unlike individuals from $A$. prolifera, all of which were heterozygous for the two loci, indicating that they are first-generation $\left(\mathrm{F}_{1}\right)$ hybrids. Mitochondrial data revealed that some genetic mixing does occur between the hybrid and its parents, but at a low level, thereby confirming that $A$. prolifgeneral, $\mathrm{F}_{1}$ hybrids - mules, for example - do not have much evolutionary potential as they cannot reproduce sexually. This is essentially true of A. prolifera as well. However, as they reproduce asexually by cloning themselves, these hybrid corals are virtually immortal, and endow the genus with many forms - a diversity that depends on the hybridization events themselves, on rare backcrosses with the parents and, curiously, on which parent provided the egg.

Corals have therefore found a potentially common way to generate ecological diversity that doesn't involve speciation. Just how common "immortal mules" such as A. prolifera are remains to be seen. ORIGINAL RESEARCH PAPER Vollmer, S. V. \& ORIGINAL RESEARCH PAPER Vollmer, S. V. \&
Palumbi, S. R. Hybridization and the evolution of reef coral diversity. Science 296, 2023-2025 (2002)

\section{WEB SITE}

Steve Palumbi's lab:

http://www.oeb.harvard.edu/palumbi era arises from recent hybridization. In

\section{(29) References and links}

\section{IN BRIEF}

\section{MOLECULAR EVOLUTION}

Identifying genes of agronomic importance in maize by screening microsatellites for evidence of selection during domestication.

Vigouroux, Y. et al. Proc. Natl Acad. Sci. USA 8 July 2002 (10.1073/pnas.112324299)

The domestication of cereals was accompanied by selection for improved agronomic and nutritional qualities; this paper reports a molecular genetic approach for identifying the genes underlying these important traits. Five hundred and one genes were screened for reduced microsatellite genetic diversity in domesticated maize compared with its wild ancestor. This work shows that evolutionarily important genes can be defined molecularly without previous knowledge of their phenotypic functions.

\section{MOUSE MODELS}

Removing the vertebrate-specific TBP $N$ terminus disrupts placental $\beta 2 \mathrm{~m}$-dependent interactions with the maternal immune system.

Hobbs, N. K. et al. Cell 110, 43-45 (2002)

Studying how modifications to the basic transcription machinery control complex gene-expression patterns led Hobbs et al. to an unexpected discovery. They generated mice that carried a TATA-binding protein (TBP) with a deletion of its vertebratespecific amino-terminus. Most mice died in mid-gestation, but this effect could be rescued by wild-type placenta or if the mothers were severely immunocompromised, especially if they lacked $\beta 2$-microglobulin $(\beta 2 \mathrm{~m})$. The authors propose that TBP transcriptionally regulates a $\beta 2 \mathrm{~m}$-dependent process involved in placental evasion of a maternal immune response.

\section{RECOMBINATION}

Reciprocal crossover asymmetry and meiotic drive in a human recombination hotspot.

Jeffreys, A. J. \& Neumann, R. Nature Genet. 31, 267-271 (2002)

Crossover clustering and rapid decay of linkage disequilibrium in the Xp/Yp pseudoautosomal gene SHOX.

May, C. A. et al. Nature Genet. 31, 272-275 (2002)

In these papers, Alec Jeffrey's group analysed recombination events in human sperm DNA. In the first, the authors report a SNP at the centre of a recombination hot spot in the major histocompatibility complex that suppresses recombination by delaying crossover initiation. Crossovers in heterozygotes are consequently accompanied by gene conversion, resulting in the overtransmission of the recombination-suppressing allele. Computer simulations show that such alleles might become fixed by meiotic drive, leading to the extinction of human recombination hot spots The accompanying paper looked at recombination patterns in the pseudoautosomal region PAR1. This high-resolution analysis unexpectedly shows that crossovers cluster into a recombination hot spot similar to autosomal hot spots, indicating that PAR1 might be useful for future studies of recombination. 